\title{
Cosmic strings confront COBE
}

\author{
B. Allen ${ }^{1,2}$, R.R. Caldwell ${ }^{3}$, E.P.S. Shellard ${ }^{2,3}$, \\ A. Stebbins ${ }^{4}$ and $S$. Veeraraghavan ${ }^{5}$ \\ 1 Department of Physics \\ University of Wisconsin \\ Milwaukee, Wisconsin 53201 \\ 2 Isaac Newton Institute \\ Clarkson Rd, Cambridge CB3 OEH, U.K. \\ 3 Department of Applied Mathernatics and Theoretical Physics \\ University of Cambridge, Silver Street, Cambridge, CB3 9EW, U.K. \\ 4 NASA/Fermilab Astrophysics Center \\ Fermi National Accelerator Laboratory \\ P. O. Box 500, Batavia, Illinois 60510 \\ 5 Goddard Space Flight Center, \\ Greenbelt, Maryland 20771
}

\begin{abstract}
We investigate large-angle anisotropies in the cosmic microwave radiation background which are induced by cosmic strings. We numerically evolve a string network from a redshift $Z=100$ to the present day and we use this data to generate a full-sky temperature map using sophisticated Green's function methods. Based on a limited number of realizations, we have compared the results of our simulations with the observations of the COBE-DMR experiment, demonstrating broad consistency. We have obtained a preliminary normalization for the string mass-per-unit-length $\mu$ in the cosmic string scenario.
\end{abstract}

\section{Introduction}

Cosmic strings are relics from a hot dense phase of the early universe [1]. They have been invoked to solve a variety of cosmological enigmas, including the origin of the primordial perturbations necessary to seed galaxy formation [2]. Their properties and cosmological implications have been actively investigated for some considerable time [3]. The understanding of the effects of superheavy cosmic strings in an expanding universe can be summarized as follows: On large scales close to the horizon size, long wiggly strings sweep out wakes in the cosmological fluid, leaving overdense regions which may grow to form galaxies and clusters $[4,5]$. On small scales, small loops are chopped off and ejected from the long strings. These loops then contract and expand under their own tension, radiating gravitational waves $[6,7]$. Processes on both large and small scales contribute to the gross features of the cosmic string scenario and the associated observational signatures. It is important to note that the understanding of the cosmological implications of strings has undergone some evolution, however, 
these developments have not been due to modifications of the underlying model; no 'adjustment' of parameters has occurred. Rather, progress derives from the improving sophistication of the analytic and numerical techniques applied to the difficult non-linear study of cosmic strings.

In the cosmic string scenario for the formation of large scale structure, the cosmological perturbations in the density and microwave background radiation (MBR) are induced by the gravitational fields of the cosmic strings. Present estimates of the only free parameter of the model, $\mu$, the mass-per-unit-length, suggest $G \mu / c^{2} \sim 1-4 \times 10^{-6}[6-10]$. That is, for $\mu$ within this approximate range of values, cosmic strings produce a spectrum of density and MBR fluctuations which appear to be in rough agreement with observations. Here we examine the large-scale MBR anisotropies produced by strings more carefully than has been done previously.

We may make several preliminary observations regarding the properties of the fluctuations induced in the MBR by cosmic strings. It is well understood that a single, moving, cosmic string produces a non-Gaussian, discontinuous temperature shift on a field of photons passing by the string [11]. On large angular scales on the celestial sphere, however, the cumulative effect of the many cosmic strings present between the surface of last scattering and the observer will render less apparent the features of individual strings. Indeed, the effects of individual strings are expected to be identifiable only on smaller angular scales on the order of arcminutes $[12,13]$. Additional anisotropies will also arise from the density perturbations induced in the baryons and dark matter by the strings. For example, the spectrum of anisotropies will exhibit the same type of 'Doppler peak' as do other models, although the amplitude may be different [14]. However, these small-angle effects are not incorporated in the present study.

Here we consider the large-scale anisotropy of the MBR by computing the large-angle temperature field on the celestial sphere induced by different realizations of a numerical simulation of cosmic strings. This work is specifically designed for direct comparison with the rms temperature fluctuations found by the COBE-DMR experiment [15].

\section{Methodology}

\subsection{Cosmic string simulation}

The cosmic string simulation evolves a network of strings which lie within the past light cone of an observer, from some early time to the present. At each timestep in this interval the code updates the positions and velocities of the strings, which are discretized as a linear sequence of 'segments'. From the positions, velocities, and lengths of the segments we can compute the string stressenergy tensor, $\Theta_{\mu \nu}$, which is used to calculate the anisotropy as described below.

The string simulation is a modified version of the Allen-Shellard code, described in detail in ref. [16]. The code has been modified to permit an $\Omega=1$ matter-dominated FRW universe to undergo a much longer period of expansion 
than in previous work. This is necessary in order to follow the evolution of the cosmic strings over a sufficiently large redshift range. We have altered the code to maintain only a fixed number of cosmic string segments per horizon length, consistent with the angular resolution of the anisotropy calculation. The simulations used here have been evolved from a redshift of $Z=100$ to the present, which may be sufficient to include anisotropies produced on scales to which COBE is sensitive.

The first method we have applied to maintain a fixed level of resolution is fairly straightforward. Neighbouring pairs of sufficiently short cosmic string segments are combined by converting the adjacent pairs into a single new segment. The conditions we choose to achieve this come as close as possible to satisfying the requirements of both energy and momentum conservation, though only the former is exact. We have tested this procedure by monitoring the energy and rms velocity along long strings on different length scales. With a few caveats, we find that the segment-joined, fixed-resolution strings satisfactorily approximate the behaviour of high resolution cosmic strings. The actual degree to which segmentjoined strings approximate 'realistic' cosmic strings is the subject of continuing quantitative investigation and will be addressed in a future publication [19].

\subsection{Computation of the temperature field}

Cosmic strings contribute only a small fraction of the cosmological density parameter to the cosmological fluid. We may estimate that $\Omega_{\text {strings }} \sim(2 \pi / 3) A G \mu / c^{2}$ $10^{-4}$ where $A \sim 30$ is the average number of long strings present in a horizon volume. Consequently, the cosmic string gravitational field will induce only small perturbations in the surrounding matter and metric. The perturbations in the matter will grow via gravitational instability, and may produce the structures we observe today in the universe. The metric perturbations induced directly by the strings and indirectly via matter perturbations will remain weak. The photons travelling through these time-varying weak gravitational fields, however, will gain or lose energy via the Sachs-Wolfe effect [17], ultimately leading to the anisotropy observed in the MBR.

One may use linear theory to express the anisotropy pattern in terms of the stress-energy of the string:

$$
\left(\frac{\delta T}{T}\right)(\theta, \phi)_{S}=\int d^{4} x G_{S}^{\mu \nu} \Theta_{\mu \nu} .
$$

Here, $S$ indicates the contribution by strings. To compute the Green's functions $G_{S}^{\mu \nu}$ one first calculates the metric perturbations in terms of $\Theta_{\mu \nu}$, and then inserts this integral solution into the Sachs-Wolfe formula as shown in ref. [18]. These Green's functions have support on and within the past light cone of the observer.

Now, because the cosmic strings were formed out of the background cosmological fluid via the Kibble mechanism at some early time [1], the energy and momentum in the strings creates a corresponding deficit in the background 
fluid. Subsequently, this anti-correlation between the energy-momentum of the strings and the energy-momentum of the rest of the matter/radiation will be maintained at all later times. We incorporate this compensation by including matter perturbations in the initial data. The resulting contribution is added to the temperature field (1) and it makes a significant correction to the anisotropy at large angular scales.

We perform the integral described in equation (1) by summing the contributions of each discretized string segment at each time step. The temperature pattern is itself discretized by constructing a grid of 6144 pixels on the celestial sphere, each approximately $3^{\circ} \times 3^{\circ}$ in size (as in the COBE-DMR experiment). This formalism may appear to be cumbersome, but the effective dimensionality of the integrals may be reduced because of a number of symmetries. The code has been tested by comparing the computed anisotropy pattern for simple string configurations with known analytic results. We find good agreement and further tests are currently being pursued. A more complete description of our numerical methods, analytic tests and simulation results is in preparation [19].

\section{Preliminary results}

The output of the numerical simulation is the (discretized) temperature field on the celestial sphere, $(\Delta T / T)(\theta, \phi)$. The anisotropy pattern seen by a single observer in one realization of a $z=100$ string network is shown in fig. 1 using the equal area Hammer-Aitoff projection. Monopole and dipole moments have been subtracted from the map and no smoothing has been performed, so the individual pixels are identifiable. There are no particularly striking features in fig. 1 which has a rather gaussian appearance. It should be noted, however, that these are preliminary results and we are still investigating the possibility of a variety of systematic errors.

Using this temperature field we may also compute the power spectrum of the MBR fluctuations, defined by

$$
C_{l}=\frac{1}{2 l+1} \sum_{m=-l}^{l}\left|a_{l m}\right|^{2} \quad \frac{\Delta T}{T}(\theta, \phi)=\sum_{l=0}^{\infty} \sum_{m=-l}^{l} a_{l m} Y_{l m}(\theta, \phi) .
$$

In figure 2 we present the anisotropy spectrum, $\left[l(l+1) C_{l} / 2 \pi\right]^{1 / 2} /\left(G \mu / c^{2}\right)$, obtained from two realizations. Some difference between the two spectra is expected due to 'cosmic variance'. The fall off at large $l$ is at least partly due to the discretization of the temperature field on the celestial sphere. There also appears to be a fall off at low multipole moments, although not as steeply as had been previously predicted $[8,10]$.

Averaging the rms temperature from two realizations we find

$$
(\Delta T)_{\text {r.m.s. }} \approx 9 G \mu / c^{2} \text {. }
$$

We can compare this rms temperature anisotropy directly with the results obtained from the two-year COBE-DMR data [15],

$$
(\Delta T)_{\text {r.m.s. }}^{\text {DMR }}=(30.5 \pm 2.7) \times 10^{-6} \mathrm{~K}, \quad T=2.7 \mathrm{~K},
$$




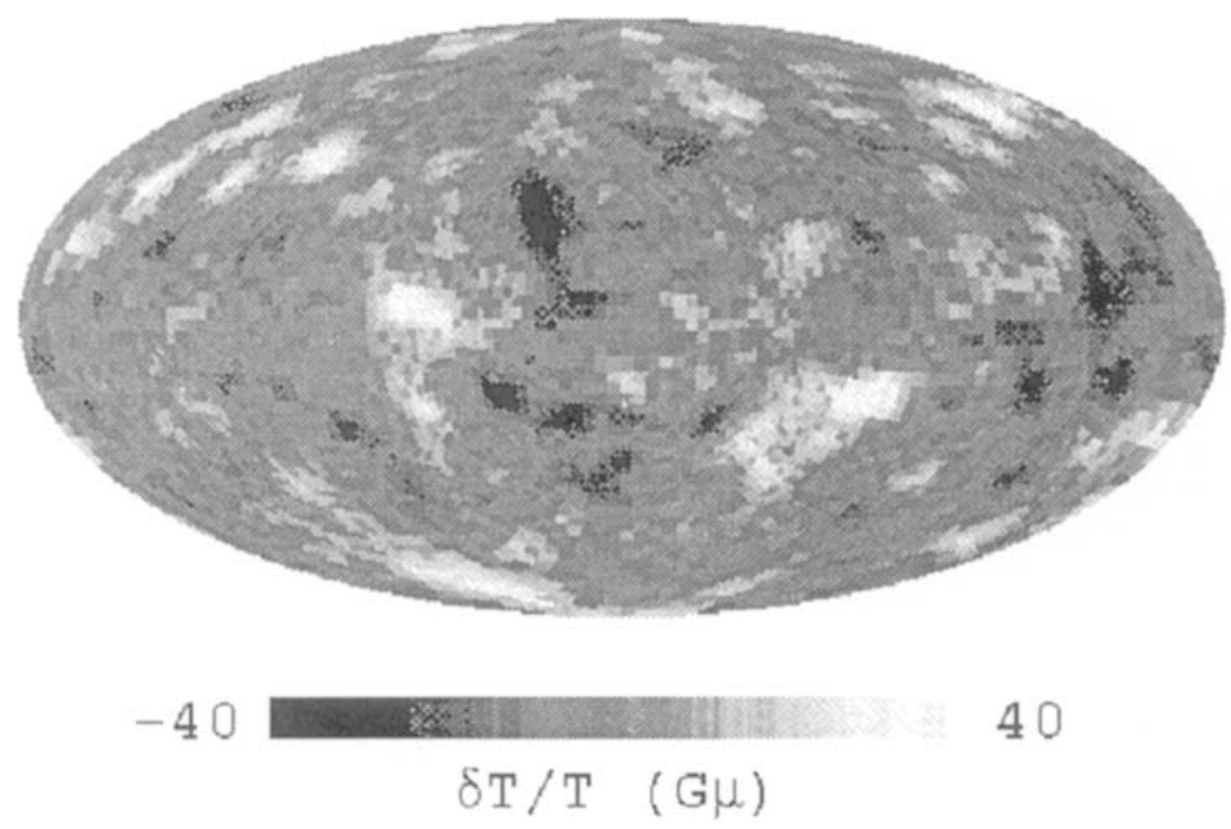

Fig. 1. The anisotropy pattern induced by cosmic strings, for one observer location in a cosmic string simulation, as described in the text. The monopole and dipole moments have been subtracted.

which yields the overall string normalization,

$$
\frac{G \mu}{c^{2}} \approx 1.3 \times 10^{-6} .
$$

At present we can put no meaningful error bars on this preliminary result, but we note the consistency with a previous extrapolated estimate using the COBE data [8].

\section{Discussion}

We have presented some preliminary results from a numerical simulation of the MBR anisotropy induced by cosmic strings. With an appropriate choice of the string parameter $\mu$, these anisotropies are consistent with the MBR anisotropies 


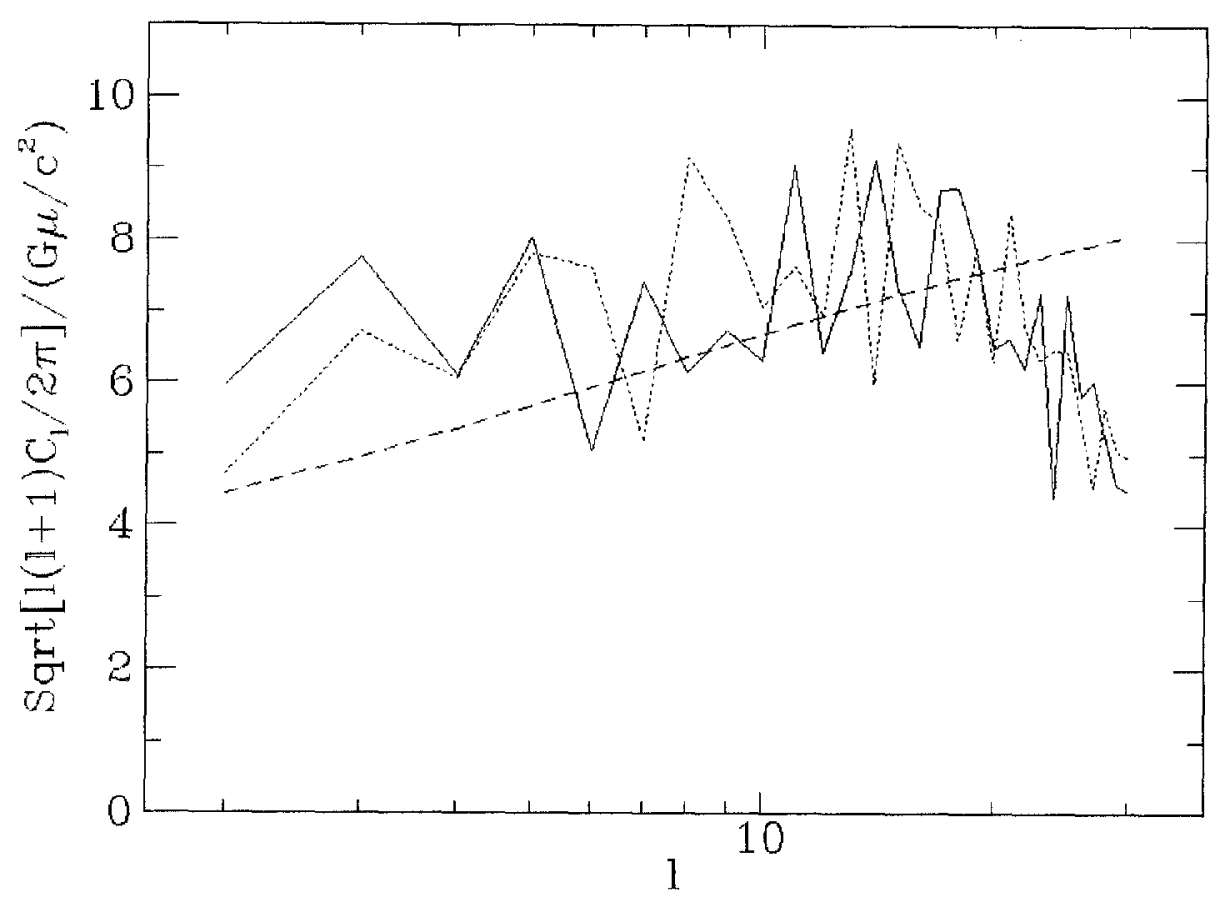

Fig. 2. The power spectrum of anisotropies, determined from the results of the numerical simulation. The solid and dotted lines are for two different observers. The fall off in the spectrum at large $l$ is at least partially due to the discretization of the temperature field on the celestial sphere. The amplitude of the spectrum is normalized by the cosmic string mass-per-unit-length $\mu$. Also shown is a model for the power spectrum given in $[8]$.

observed by the COBE-DMR experiment. Because of the sophistication of the string evolution and the full-sky treatment, this work should ultimately provide the first definitive normalization of the cosmic string scenario. It already broadly confirms other estimates of MBR anisotropies from strings $[8,13]$, and further analysis of our techniques and results will allow us to better quantify the uncertainties in this calculation [19]. In the near future this work will also quantify the angular spectral index, the degree of non-gaussianity, and the significance of cosmic variance. With this overall normalization, cosmic strings will then have to be judged against predictions for small-angle MBR anisotropies and structure formation. In explaining the COBE-DMR anisotropies, inflationary models appear to be confronted with at least one viable alternative scenario. 


\section{Acknowledgements}

The work of BA is supported by the NSF through grant no. PHY-91-05935. RRC and EPS are funded by PPARC through grant no. GR/H71550. AS is supported by NASA through grant no. NAGW-2381. SV is supported by the National Research Council.

\section{References}

1. T.W.B. Kibble, 'Some implications of cosmological phase transitions', Phys. Rep. 67, 183 (1980).

2. Ya.B. Zeldovich, 'Cosmological fluctuations produced near a singularity', MNRAS 192, 663 (1980); A. Vilenkin, 'Cosmological density fluctuations produced by vacuum strings', Phys. Rev. Lett. 46, 1169 (1981).

3. See, for example: A. Vilenkin \& E.P.S. Shellard, Cosmic strings and other topological defects (Cambridge, 1994).

4. J. Silk \& A. Vilenkin, 'Cosmic strings and galaxy formation', Phys. Rev. Lett. 53, 1700 (1984); T. Vachaspati, 'Cosmic strings and the large-scale structure of the universe', Phys. Rev. Lett. 57, 1655 (1986); A. Stebbins, S. Veeraraghavan, R. Brandenberger, J. Silk \& N. Turok, 'Cosmic string wakes', Ap. J. 322, 1 (1987); R. Brandenberger, L. Perivolaropoulos \& A. Stebbins, 'Cosmic strings, hot dark matter and the large scale structure of the universe', Int. J. Mod. Phys. A 5, 1633 (1990).

5. T. Vachaspati \& A. Vilenkin, 'Large scale structure from wiggly cosmic strings', Phys. Rev. Lett. 67, 1057 (1992); D. Vollick, 'Small scale structure on cosmic strings and galaxy formation', Phys. Rev. D 45, 1884 (1992).

6. A. Vilenkin, 'Gravitational radiation from cosmic strings', Phys. Lett. 107B, 47 (1981).

7. R.R. Caldwell \& B. Allen, 'Cosmological constraints on cosmic string gravitational radiation', Phys. Rev. D45, 3447 (1992); R.R. Caldwell, 'The current status of observational constraints on cosmic strings', Fermilab preprint 196-A (1993).

8. D. Bennett, A. Stebbins, \& F.R. Bouchet, 'The implications of the COBEDMR results for cosmic strings', Ap. J. Lett. 399, L5 (1992).

9. A. Albrecht \& A. Stebbins, 'Perturbations from cosmic strings in cold dark matter', Phys. Rev. Lett. 68, 2121 (1992); A. Albrecht \& A. Stebbins, 'Cosmic string with a massive light neutrino', Phys. Rev. Lett. 69, 2615 (1992).

10. L. Perivolaropoulos, 'COBE vs. cosmic strings: an analytical model', Phys. Lett. 298, 305 (1993).

11. N. Kaiser \& A. Stebbins, 'Microwave anisotropy due to cosmic strings', $\mathrm{Na}$ ture 310, 391 (1984).

12. R. Moessner, L. Perivolaropoulos, \& R. Brandenberger, 'A cosmic string specific signature on the cosmic microwave background', Brown preprint HET-911 (1993). 
13. D. Coulson, P. Ferreira, P. Graham, \& N. Turok, 'Microwave anisotropies from cosmic defects', Nature 368, 27 (1994).

14. L. Perivolaropoulos, 'Spectral analysis of microwave background perturbations induced by cosmic strings', CfA preprint 3796 (1994).

15. C. L. Bennett, et al., Cosmic temperature fluctuations from two years of COBE DMR observations, COBE preprint 94-01 (1994).

16. B. Allen \& E.P.S. Shellard, 'Cosmic string evolution: a numerical simulation', Phys. Rev. Lett. 64, 119 (1990); E.P.S. Shellard \& B. Allen, 'On the evolution of cosmic strings', in Formation and evolution of cosmic strings, G.W. Gibbons, S.W. Hawking \& T. Vachaspati, eds. (Cambridge, 1990).

17. R. Sachs \& A. Wolfe, 'Perturbations of a cosmological model and angular variations of the microwave background', Ap. J. 147, 73 (1967).

18. S. Veeraraghavan \& A. Stebbins, 'Causal compensated perturbations in cosmology', Ap. J. 365, 37 (1990); S. Veeraraghavan \& A. Stebbins, in preparation (1994).

19. B. Allen, R.R. Caldwell, E.P.S. Shellard, A. Stebbins \& S. Veeraraghavan, 'Large scale CMBR anisotropies induced by cosmic strings', in preparation (1994). 